\title{
Channel sharing utility function of power control game in cognitive femtocell network
}

\author{
Anggun Fitrian Isnawati ${ }^{1}$, Risanuri Hidayat ${ }^{2}$, Selo Sulistyo $^{3}$, I Wayan Mustika ${ }^{4}$ \\ ${ }^{1}$ Department of Telecommunication Engineering, Institut Teknologi Telkom Purwokerto, Indonesia \\ ${ }^{2,3,4}$ Department of Electrical and Information Technology, Universitas Gadjah Mada (UGM), Yogyakarta, Indonesia
}

\begin{tabular}{l} 
Article Info \\
\hline Article history: \\
Received Mar 27, 2019 \\
Revised May 7, 2019 \\
Accepted May 15, 2019 \\
\hline
\end{tabular}

Keywords:

Channel sharing factor Cognitive femtocell Game theory Power control game Utility function

\begin{abstract}
The use of sharing channel simultaneously has become the trend in telecommunication technology particularly in network with distributed users as the allocation of frequency spectrum becomes more crowded. The proposed power control method in cognitive femtocell network is based on game theory (commonly known as power control game, PCG). This method uses utility function formula as the goal of game theory for power strategy in power update process. Utility function formula of Proposed PCG includes channel sharing factor that aimed to accommodate the system requirement of channel sharing. The result showed that the implementation of channel factor is inversely proportional to the signal to noise and interference ratio (SINR) and transmit power, but proportional to utility function. In comparison of user transmit power and SINR with two other methods, can also be conclude that Proposed PCG was able to achieve SINR of $5.49 \mathrm{~dB}$ and above the SINR target $5 \mathrm{~dB}$, while the Thalabani (TB) and Koskie-Gajic (KG) were only able to achieve respective SINR of $4.87 \mathrm{~dB}$ and $4.98 \mathrm{~dB}$. It can be concluded that the Proposed PCG was better in achieving the SINR target. It means that the quality of service in this system can be fulfilled properly.
\end{abstract}

Copyright $(2019$ Institute of Advanced Engineering and Science. All rights reserved.

\section{Corresponding Author:}

Anggun Fitrian Isnawati, Department of Telecommunication Engineering, Faculty of Telecommunication and Electrical Engineering, Institut Teknologi Telkom Purwokerto, Indonesia. Email: anggun@ittelkom-pwt.ac.id

\section{INTRODUCTION}

In the developing telecommunication era, cellular network with centralized power control creates a new dilemma as it has better performance than distributed power control network and it is able to generate optimum solution efficiently with low communication cost and computation requirement in the small-scale system. Yet on the other hand, as the size of the network grows and the number of cellular users became uncertain, centralized algorithm is now considered ineffective. The challenge of developing distributed algorithm is to create an algorithm that is able to work independently, without global information and centralized coordination. Distributed power control could avoid bottleneck effect of centralized power control and is able to improve reliability by diminishing the failure effect in central station. Therefore, the implementation of this shows some promises [1].

Developed distributed power control includes distributed power control (DPC) method and game theoretical power control or as commonly known as power control game (PCG). PCG is a power control based on game theory approach by proposing a specific utility function. By creating the utility function that accommodates SINR target, the iteration process could be reduced in the function. SINR target is accommodated in utility function to obtain the required power to meet the intended SINR target. The result of implementing utility function tends to surpass the SINR target, meanwhile DPC method only able to reach the SINR target without exceeding it [2]. Utility could be translated as improvement of throughput and 
network capacity based on power limitation and interference total by user's ability to determine strategy in competing with other user to obtain better power control [3].

Scheme of power control has been studied extensively to minimalize interference and ensure the quality of service (QoS) requirements are met [3], create green wireless communication by resolving QoS trade-off and improving energy efficiency [4 - 6]. Studies of power control by implementing game theory are also aimed to improve the convergence rate (Nash Equilibrium) [4 - 7] and low complexity [8]. Many simple algorithms are able to be implemented in distributed power control scheme, yet they have low convergence rate and low power utilization ratio. Hence, these algorithms are difficult to be accommodated in dynamic network environment. Other studies are able to improve convergence rate and power utilization ratio; however, they do not meet the requirement to be classified as smart network. Therefore, this study proposed distributed power control algorithm based on efficient game theory with high convergence rate in stable condition [4]. Moreover, studies of power control in femtocell network are classified based on channel use, with single channel and multi-channel where each user can accommodate his power through various frequency channels [9]. Application of multi-channel in femtocell network is also able to improve utility function, minimize interference, increase throughput, and improve convergence rate (iteration efficiency).

Previous studies have not shown the effect of channel sharing factor parameter on utility function. Hence, this study introduced channel sharing factor and analyze its application and its effect on user's convergence rate, user transmitted power, utility function improvement, and ability to meet SINR target as parameter of QoS of a system.

\section{RESEARCH METHOD}

\subsection{System model}

Figure 1 displays the user and channel model in cognitive femtocell network. In this study, the user is a pair of femto user equipment (FUE) as transmitter (Tx) and femto access point (FAP) as receiver (Rx). User data generation influenced by the number of femtocell used in co-tier network topology. Since users in co-tier topology is a pair, the number of femtocells shows the number of generated user in multi-channel system, as illustrated in Figure 2.

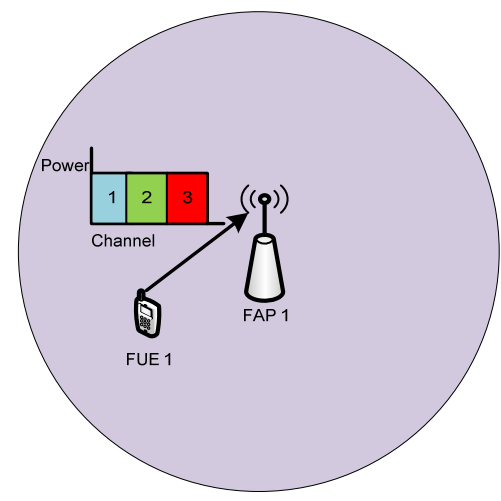

Figure 1. User and channel model in femtocell network

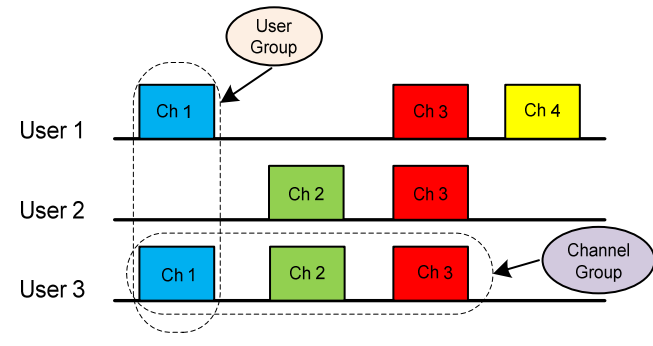

Figure 2. Channel division scheme in femtocell users

Channel division of existing users can be observed in Figure 2. The focus of channel sharing is user group as each user share his channel with others. Figure 2 shows a condition where channel-1 is shared by user-1 and user-3, thus user-1 channel- 1 can be denoted as $\mathrm{U} 1 \mathrm{C} 1$, and user- 3 channel-1 as $\mathrm{U} 3 \mathrm{C} 1$.

\subsection{Utility function of $P C G$}

Utility function of PCG is determined by factors influencing system performance such as SINR target, achieved SINR user, maximum number of users allowed, user power, and other parameters that determine the quality of service (QoS). Utility function represents player's preference, where if player $i$ prefer $a \epsilon S$ strategy to $b \in S$ strategy, then $u_{i}(a)>u_{i}(b)$. which are:

In general, utility function can be classified into two functions with their own characteristics, 
a. Reward Function

- Comes in the form of SINR or obtained bit capacity by the change of power strategy

- Tends to increase utility (positively)

- The power is directly proportional to the reward value thus improving the total value of utility function

b. Pricing Function

- Could come in the form of interference caused by power strategy choice

- Tends to decrease utility (negatively)

- The power is directly proportional to the pricing value thus the total value of utility function is decreased.

Determination of utility and pricing functions is used to establish user transmitted power efficiency. For example, user located near FAP must be charged with low pricing factor as low power assumption. On the contrary, user that is far from FAP must be charged with high pricing factor as high power consumption. Determination of utility function is also adjusted to predetermined proposed model and supported by other influencing factors such as channel use, SINR target determination, maximum power transmit, and other factors. The use of channel is directly proportional to power consumption, therefore in multi-channel cognitive femtocell system, the more the channel used, the more the power transmitted.

In this study, utility function is focused on each user's channel, thus every user has accumulative utility function comprised of various utility functions of each used channel. For example, a user who uses channel 1 and channel 2 then its power is accumulated in both channels. Hence, as the number of used channel increases, the required power also rises, since every channel has its own unique SINR and power that affect utility function. In this study, the proposed utility function was implemented in every user's channel, since every channel has its own unique SINR and power that affect utility function. Hence, the effect of SINR and user's power are the main factors in formulating new utility function. The formula of utility function in this study is called Proposed PCG [10]:

$$
U_{i}=a_{i} p_{i}^{2}-2 b_{i} \lambda_{k} \gamma_{i}+c_{i}\left(\gamma_{i}^{t a r}-\gamma_{i}\right)^{2}
$$

where $a_{i}, b_{i}$ and $c_{i}$ are constants, $\lambda_{k}$ is channel sharing factor, $\gamma_{i}^{t a r}$ is SINR target, and $\gamma_{i}$ is user SINR.

In DPC method, as explained in utility function characteristic discussion $[11,12]$, when the SINR is fixed, then the increase of power will reduce the utility value to the zero level (0). This theory is in line with DPC utility function equation [13]:

$$
U_{i}=c_{i}\left(\gamma_{i}^{t a r}-\gamma_{i}\right)^{2}
$$

where $\gamma_{i}^{\text {tar }}=\gamma_{i}$ is occurred when the DPC system reached convergence condition and then $U_{i}=0$.

As power control methods in distributed system, connection between DPC and PCG methods appears in utility function formula. DPC method can be referred to determine utility function formula and power update of PCG method. Power update formula is comprised of two components, DPC component (static component) and dynamic one. Dynamic components is the difference between DPC and PCG methods. Dynamic component in power update formula also differs with pricing function component in utility function formula. Pricing function component is implemented as disincentive when user use power excessively, meanwhile dynamic component is related with power update process to measure user power value in convergence in order to obtain preferred user SINR.

Other than analysis of performance comparison of DPC and PCG methods [2], there is other analysis based on various parameters such as utility function of determining Nash Equilibrium. This analysis does not need comparison, but it requires the proof of utility function characteristic of DPC and PCG methods. Since the objective of game theory is maximizing player's utility function based on strategy implementation, the utility value in PCG should increase along with the power increase, as reported by [14]. This fact is what differ PCG method with DPC. The utility value could also decrease due to several factors such as, increase of the number of users used shared channel [15] and also distance between user and FAP $[16,17]$

\subsection{Power update formula}

The power update equation is used by the user to independently update the power based on the received SINR. This power update equation is obtained from the previous equation of the specified utility function $\left(U_{i}\right)$ which is derived to user power $\left(p_{i}\right)$. The iteration process based on the power update equation will produce optimal power. Mathematically, it is formulated as [18]: 


$$
\frac{d U_{i}}{d p_{i}}=0
$$

Based on (3), the power update formula can be obtained from (1) for the analysis of transmit power in the Proposed PCG method as follows [10]:

$$
p_{i}^{(t+1)}=\left(\gamma_{i}^{t a r}+\lambda_{k}\right) \frac{p_{i}^{(t)}}{\gamma_{i}^{(t)}}-\frac{b_{i}\left(p_{i}^{(t)}\right)^{3}}{c_{i}\left(\gamma_{i}^{(t)}\right)^{2}}
$$

In the performance analysis process, the Proposed PCG method will be compared with the other two PCG methods, the Koskie Gajic (KG) [13] and the Thalabani (TB) [19] which are shown in (5) and (6):

$$
\begin{aligned}
& p_{i}^{(t+1)}=\gamma_{i}^{t a r}\left(\frac{p_{i}^{(t)}}{\gamma_{i}^{(t)}}\right)-\frac{a_{i}}{c_{i}}\left(\frac{p_{i}^{(t)}}{\gamma_{i}^{(t)}}\right)^{2} \\
& p_{i}^{(t+1)}=\left(\frac{p_{i}^{(t)}}{\gamma_{i}^{(t)}} \gamma_{i}^{t a r}\right)-\left(\frac{p_{i}^{(t)}}{\gamma_{i}^{(t)}} \Delta_{t a r, i}^{(t)}\right)-\frac{b_{i}\left(p_{i}^{(t)}\right)^{3}}{c_{i}\left(\gamma_{i}^{(t)}\right)^{2}}
\end{aligned}
$$

\subsection{Channel sharing factor}

This study introduces channel sharing factor $\left(\lambda_{k}\right)$ that appears in equation of Proposed PCG utility function (1) and power update (4). This factor is formulated as:

$$
\lambda_{k}=\frac{1}{k}=\frac{1}{2^{r}}
$$

If $k=2^{r}=1 / \lambda_{k}$ then $r=\log _{2} k=-\log _{2} \lambda_{k}$, with $\lambda_{k}$ is channel sharing factor, $r$ is channel factor and $k$ is channel size (resource block, RB). Channel sharing factor shows the maximum number of user that can be accommodated in each channel while channel factor $(r)$ is the value related with channel size $(k)$ used in system that inversely proportional to $\lambda_{k}$.

\section{RESULTS AND DISCUSSION}

\subsection{Characteristics of utility function}

Figure 3 shows that in DPC, characteristic of utility function decreased to nearly reach zero value. This result is in line with (2), where it states that in convergence, SINR received by user is equal to SINR target, thus the value of utility function is zero. Meanwhile, PCG had contrary characteristic as illustrated in Figure 4.

As displayed in Figure 4, Proposed PCG method also had increasing utility value as the power increased. The change of power increase only occurred before the system reached Nash equilibrium (convergence).

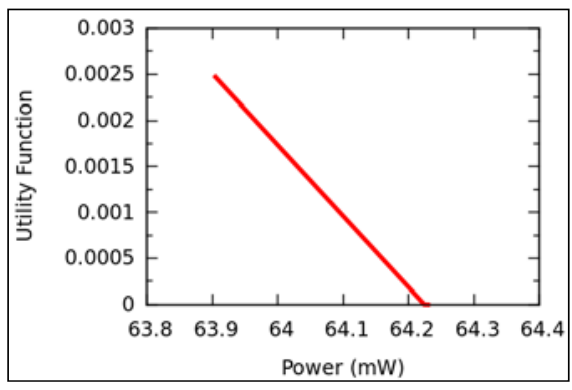

Figure 3. User's utility function in DPC method

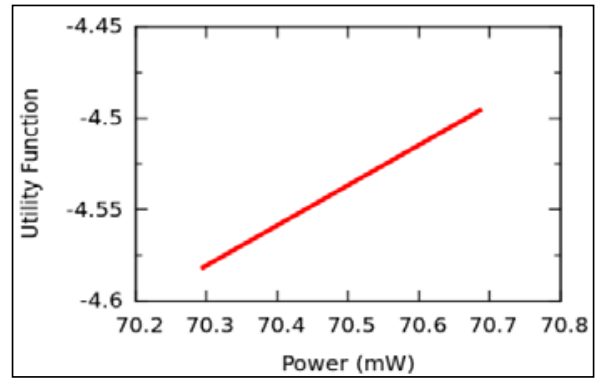

Figure 4. User's utility function in Proposed PCG method 


\subsection{Implementation of channel sharing factor}

The change of channel sharing factor $\left(\lambda_{k}\right)$ value or in utility function or power update equations was aimed to observe the effect of varied channel sharing factor $\left(\lambda_{k}\right)$ value on improving system performance in terms of transmitted power efficiency, ability to meet SINR target, or convergence rate.

Channel sharing factor $\left(\lambda_{k}\right)$ value can be set as homogeny (static) based on the average number of channel used simultaneously compared with total number of allocated channels, or adaptive (dynamic) based on each user who shares one channel compared with total number of allocated channels. Proper selection of channel sharing factor $\left(\lambda_{k}\right)$ value was expected to improve system performance based on the three parameters: SINR, power, and utility function.

As explained in (3), channel sharing factor $\left(\lambda_{k}\right)$ is inversely proportional to channel factor $(r)$, thus as the $\lambda_{k}$ value decreases, the value of $r$ rises. The decreasing value of $\lambda_{k}$ means that the maximum number of users that can be accommodated in one channel increases. Characteristic of channel sharing factor affected the change of user SINR as illustrated in Figure 5. As the value of $\lambda_{k}$ decreased, the channel factor $(r)$ increased, which means that the channel size $(k)$ increased, thus the user SINR consequently decreased to nearly reach target SINR that was $5 \mathrm{~dB}$. This result was similar with DPC characteristics. When the channel was not shared with the value of $\lambda_{k}=1$ or $r=0$, the SINR value reached the highest number of $5.999 \mathrm{~dB}$.

Figure 6 shows that as the $\lambda_{k}$ value decreased ( $r$ increased), or as the channel size $(k)$ grew, then the required power declined. This result is related to the decreased number of SINR received by user as the $r$ value rise, as illustrated in Figure 5. Moreover, Figure 7 displays the change of utility function caused by the variation of channel factor $(r)$. In this figure, as the $r$ value grew, the utility function increased to nearly reach zero value (0). This result means that the characteristic in Figure 2 is supported by Figure 7, as the variation of $\lambda_{k}$ or $r$ influences the user's utility value.

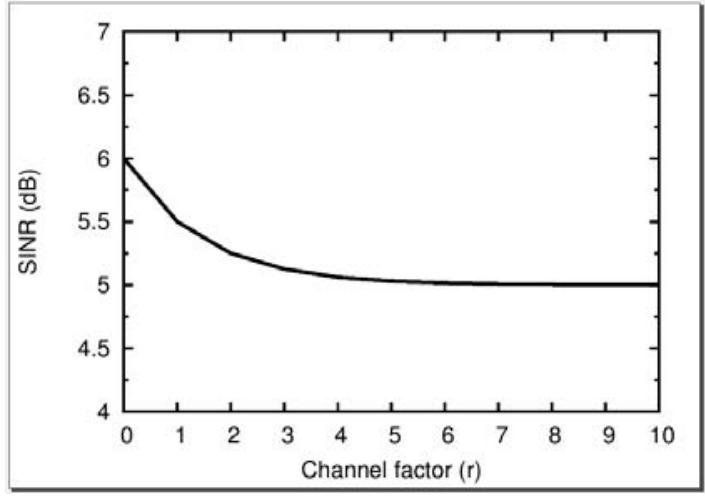

Figure 5. Change of SINR due to channel factor $(r)$ variation

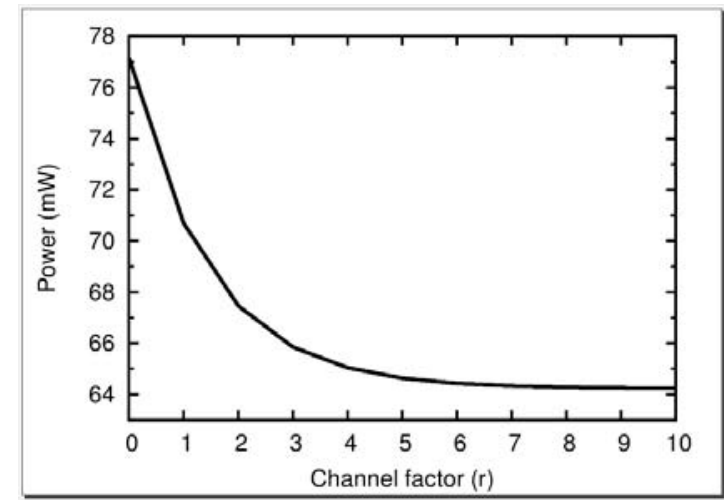

Figure 6. Change of power due to channel factor $(r)$ variation

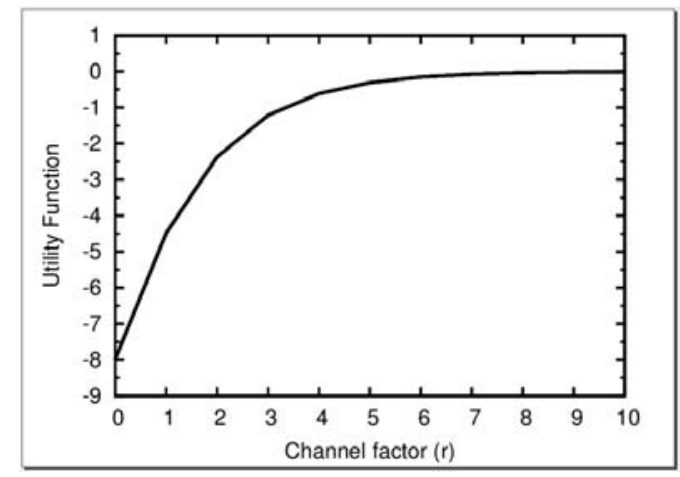

Figure 7. Change of utility function due to channel factor $(r)$ variation 


\subsection{Analysis of convergence in utility function}

Analysis of convergence in utility function is based on user iteration requirement to reach convergence. The variation of $\lambda_{k}$ influences the change of utility function value, but it does not affect the number of iteration required by user to reach convergence. Significant factor influences the number of iteration are distance among users and the number of users who share the channel.

Figure 8 illustrates characteristic of utility function when $\lambda_{k}=1$ or when only one user used the channel (not included in the real channel sharing). As can be seen in Figure 8, this utility value was higher than the previous on before reaching convergence in specific value and iteration.

This result also displayed in utility function characteristic in in Figures 9 and 10. Both figures show the characteristic of utility function when one user share the channel with another user. The value of $\lambda_{k}=1 / 2$ is the highest value of channel sharing factor as the minimum number of users who share the channel is 2 users. Meanwhile, the number of $\lambda_{k}=1 / 8$ means that there were 8 users who shared the channel.

Figure 11 shows the utility function comparison of the three values of $\lambda \mathrm{k}$. With the smaller number of $\lambda \mathrm{k}$ the resulting utility function yang value will be larger. However, this fact does not affect the number of iteration required by user to reach convergence. This can be translated as the lack of influence of channel sharing factor on user's convergence rate.

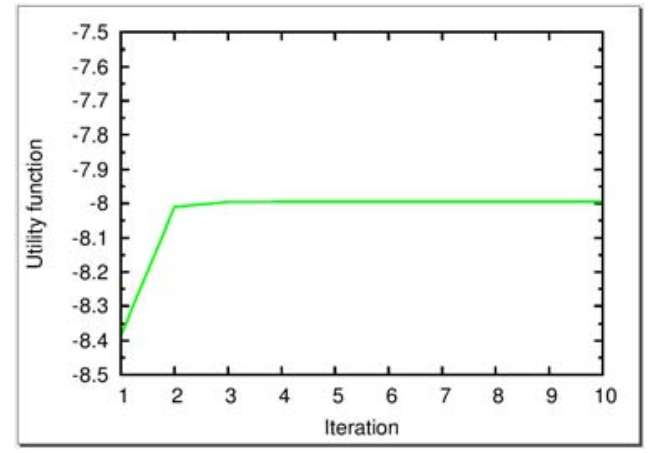

Figure 8. Utility function when $\lambda_{k}=1$

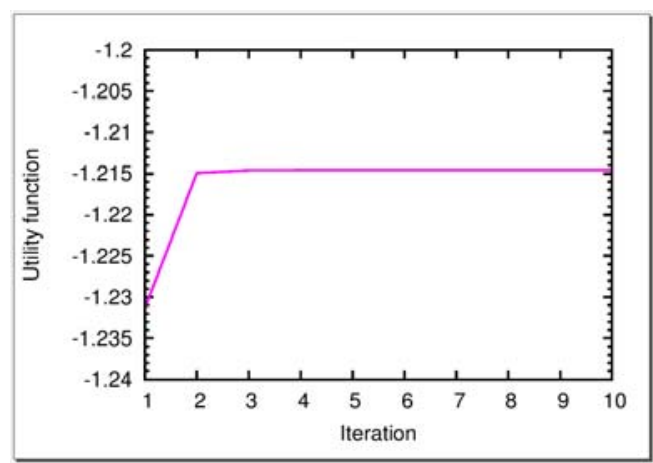

Figure 10. Utility function when $\lambda_{k}=1 / 8$

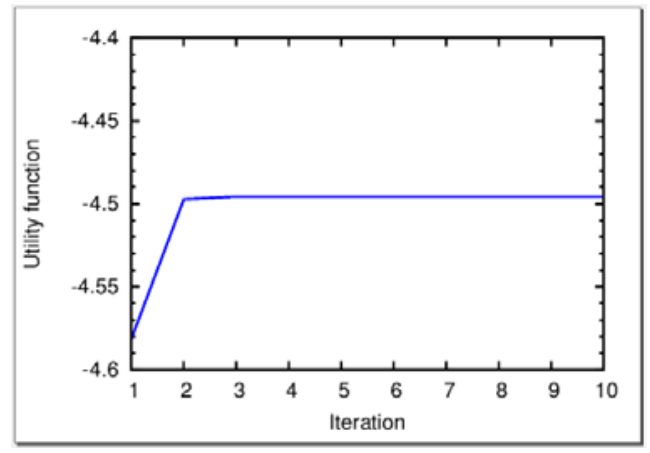

Figure 9. Utility function when $\lambda_{k}=1 / 2$

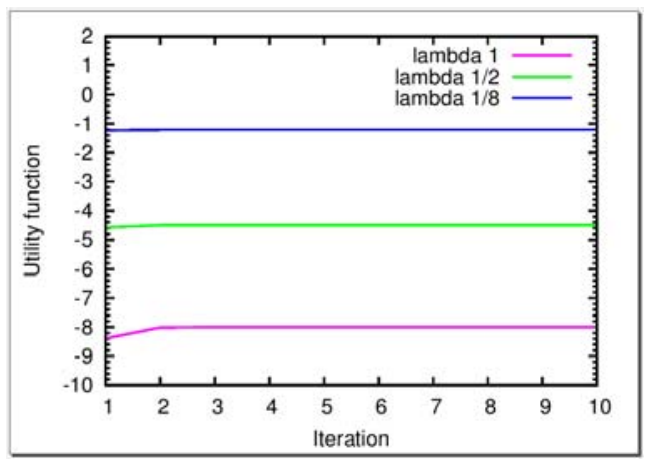

Figure 11. Utility function comparison of various $\lambda_{k}$ values

\subsection{Analysis of Transmit Power and SINR}

Figures 12 and 13 show a comparison of Proposed PCG with two other methods, Thalabani (TB) and Koskie-Gajic (KG) methods. As can be seen in Figure 12, the power usage of proposed PCG converged in a higher power, but it was used to meet the specified target SINR.

The result in Figure13 showed that the Proposed PCG was able to achieve SINR of 5.49 dB and above the SINR target $5 \mathrm{~dB}$, so that the quality of service in this system can be fulfilled properly. The results of the Thalabani (TB) and Koskie-Gajic (KG) methods have a relatively lower power but were not able to 
achieve the target SINR as shown in Figure 13. As can be seen in Figure 13, the Thalabani (TB) and KoskieGajic (KG) were only able to achieve respective SINR of $4.87 \mathrm{~dB}$ and $4.98 \mathrm{~dB}$, which were lower than the target SINR of $5 \mathrm{~dB}$, hence their quality were still below the Proposed PCG.

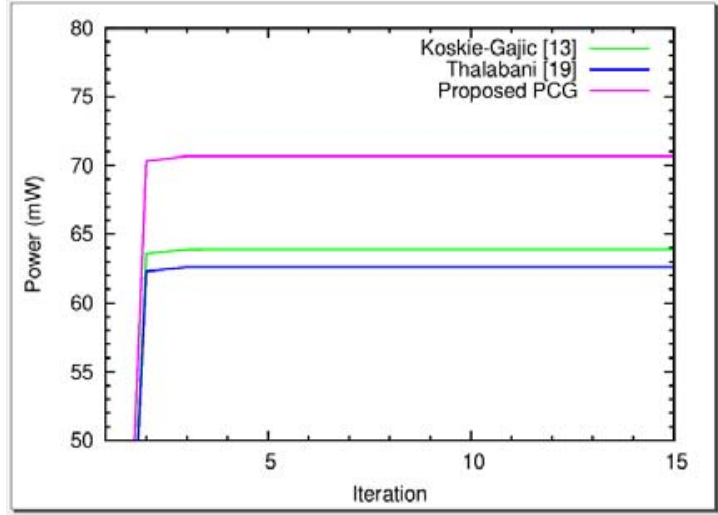

Figure 12. Comparison of user transmit power of Proposed PCG with two other methods

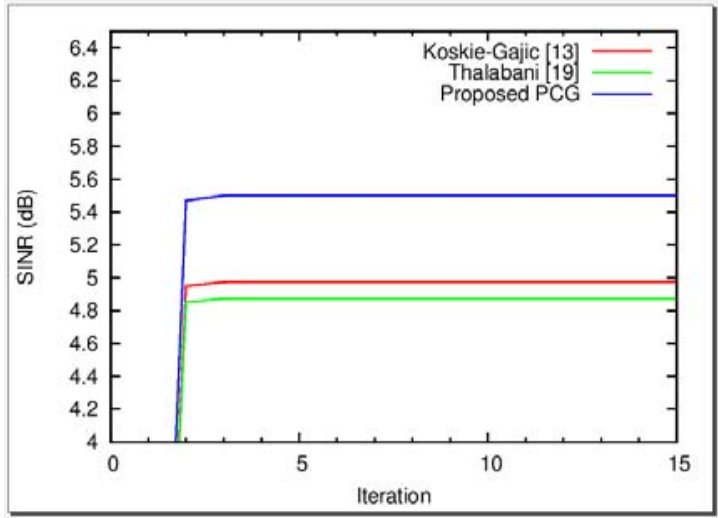

Figure 13. Comparison of user SINR of Proposed PCG with two other methods

\section{CONCLUSION}

It can be concluded from the results that as the channel sharing factor $\left(\lambda_{k}\right)$ decreases, or the channel factor $(r)$ increases, which meant that more user share the channel, the user SINR also decreases. As the value of channel sharing factor decreases, the required power also decreases as the user SINR decreases. In relation with utility function, the smaller value of channel sharing factor means that the larger number of users that can be accommodated in the channel (channel size $k$ increases), thus increasing the number of utility value. From the comparison results of user transmit power and SINR, Proposed PCG was able to achieve SINR of $5.49 \mathrm{~dB}$ and above the SINR target $5 \mathrm{~dB}$, while the Thalabani (TB) and Koskie-Gajic (KG) were only able to achieve respective SINR of $4.87 \mathrm{~dB}$ and $4.98 \mathrm{~dB}$. It can be concluded that the Proposed PCG was better in terms of achievement of the SINR target.

\section{REFERENCES}

[1] S. A. Saad, M. Ismail, and R. Nordin, "A Survey on Power Control Techniques in Femtocell Networks," $J$. Commun., vol. 8, no. 12, pp. 845-854, Dec. 2013.

[2] A. F. Isnawati, R. Hidayat, S. Sulistyo, and I. W. Mustika, "Distributed Power Control vs Power Control Game A Comparison Study of Performance in Cognitive Femtocell Network," presented at the 2017 IEEE International Conference on Applied System Innovation (ICASI), Sapporo, pp. 1841-1844, 2017.

[3] J. Duan, J. Liu, S. Leng, and Q. Wang, "A game-based power control scheme for cognitive radio networks," in Computational Problem-Solving (ICCP), International Conference on, pp. 76-79, 2012.

[4] J. Zhang, Z. Zhou, H. Yao, Y. Zhou, and K. S. Kwak, "A novel non-cooperative power control game for cognitive radio networks," in Communications and Information Technology, ISCIT 9th International Symposium on, pp. $115-118,2009$.

[5] A. J. Luah and C. K. Tan, "A Nash-based power control game for green communications via cognitive radio networks," in Sustainable Utilization and Development in Engineering and Technology (STUDENT), IEEE Conference on, pp. 164-169, 2012.

[6] B. Wang, Y. Wu, and K. J. R. Liu, "Game theory for cognitive radio networks: An overview," Comput. Netw., vol. 54, no. 14, pp. 2537-2561, Oct. 2010.

[7] Z. Junhui, Y. Tao, G. Yi, W. Jiao, and F. Lei, "Power control algorithm of cognitive radio based on noncooperative game theory," Commun. China, vol. 10, no. 11, pp. 143-154, 2013.

[8] J. Jiao, L. Jiang, and C. He, "A Novel Game Theoretic Utility Function for Power Control in Cognitive Radio Networks," presented at the Computational and Information Sciences (ICCIS), 2013 Fifth International Conference on, pp. 1553-1557, 2013.

[9] W. Li, H. Zhang, W. Zheng, T. Su, and X. Wen, "Energy-efficient power allocation with dual-utility in two-tier OFDMA femtocell networks," in Globecom Workshops (GC Wkshps), 2012 IEEE, pp. 535-540, 2012.

[10] A. F. Isnawati, R. Hidayat, S. Sulistyo, and I. W. Mustika, "A Novel Utility Function of Power Control Game in Multi-Channel Cognitive Femtocell Network,” Int. J. Commun. Netw. Inf. Secur. IJCNIS, vol. 9, no. 3, 2017. 
[11] M. Musku, A. Chronopoulos, D. Popescu, and A. Stefanescu, "A game-theoretic approach to joint rate and power control for uplink CDMA communications," IEEE Trans. Commun., vol. 58, no. 3, pp. 923-932, Mar. 2010.

[12] D. Famolari, N. Mandayam, D. Goodman, and V. Shah, "A new framework for power control in wireless data networks: Games, utility, and pricing," in Wireless Multimedia Network Technologies, Springer, pp. 289-309, 2002.

[13] S. Koskie and Z. Gajic, "A nash game algorithm for SIR-based power control in 3G wireless CDMA networks," IEEEACM Trans. Netw., vol. 13, no. 5, pp. 1017-1026, Oct. 2005.

[14] Y. A. Al-Gumaei, K. A. Noordin, A. W. Reza, and K. Dimyati, "A new power control game in two-tier femtocell networks," in Telematics and Future Generation Networks (TAFGEN), 2015 1st International Conference on, pp. 131-135, 2015.

[15] S. Buzzi and D. Saturnino, "A Game-Theoretic Approach to Energy-Efficient Power Control and Receiver Design in Cognitive CDMA Wireless Networks," IEEE J. Sel. Top. SIGNAL Process., vol. 5, no. 1, pp. 137-150, Feb. 2011.

[16] C. U. Saraydar, N. B. Mandayam, and D. Goodman, "Efficient power control via pricing in wireless data networks," Commun. IEEE Trans. On, vol. 50, no. 2, pp. 291-303, 2002.

[17] X. Wang and Q. Zhu, "Power control for cognitive radio base on game theory," in Wireless Communications, Networking and Mobile Computing, 2007. WiCom 2007. International Conference on, pp. 1256-1259, 2007.

[18] N. Omidvar and B. H. Khalaj, "A game theoretic approach for power allocation in the downlink of cognitive radio networks," in 2011 IEEE 16th International Workshop on Computer Aided Modeling and Design of Communication Links and Networks (CAMAD), pp. 158-162, 2011.

[19] A. Al Talabani, A. Nallanathan, and H. X. Nguyen, "A Novel Chaos Based Cost Function for Power Control of Cognitive Radio Networks,” IEEE Commun. Lett., vol. 19, no. 4, pp. 657-660, Apr. 2015.

\section{BIOGRAPHY OF AUTHORS}
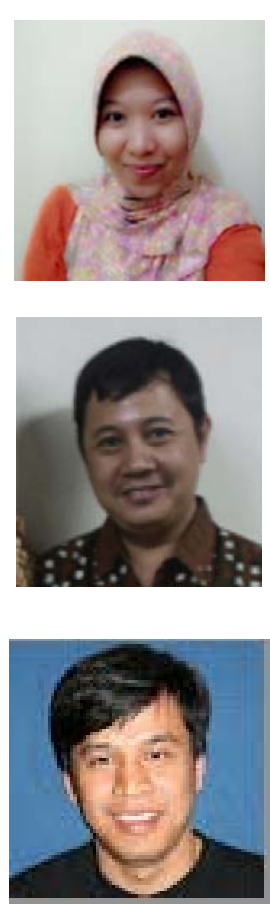

Anggun Fitrian Isnawati was born in Cilacap, Indonesia, in 1978. She received Doctoral degree in Telecommunication Engineering from Gadjah Mada University, Yogyakarta, Indonesia in 2018. She joined as a Lecturer in the Institut Teknologi Telkom Purwokerto since 2002. Her research interest is in wireless communication, signal processing, channel estimation, optical communication, game theory and cognitive radio.

Risanuri Hidayat received Master in Information and Communication Technology from Agder University College, Norway, in 2002 and Doctor in Telecommunication Engineering, King Mongkut's Institute of Technology Ladkrabang (KMITL), Bangkok, Thailand, in 2009. He is now an Associate Professor at Department of Electrical Engineering and Information Technology, Gadjah Mada University. His interest is in Signal Processing, Digital and Analog Circuit Design, Telecommunication Systems, Data Communication and Networking, Java Programming and its Applications, and also Speech and Image Recognition.

Selo Sulistyo is an Associate Professor in Information and Communication Technology at the Department of Electrical Engineering and Information Technology and also Head of System Electronics laboratory at Gadjah Mada University. His research interests including Software Certification, Software Modeling, Domain Specific Languages, Mobile application development and Software Service Engineering which relates to the Internet of Things and connected objects.

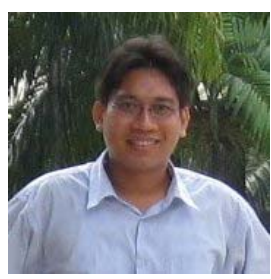

I Wayan Mustika received the B.Eng. degree in electrical engineering from Gadjah Mada University, Indonesia, in 2005, and the M.Eng degree in computer engineering from King Mongkut's Institute of Technology Ladkrabang (KMITL), Thailand, in 2008, and the Ph.D. degree in informatics from Kyoto University, Japan, in 2011. His research interests include smart systems, machine-to-machine communications, and resource management in cognitive radio and heterogeneous networks with a particular emphasis on spectrum sharing and game theory. He received the Young Researcher's Encouragement Award from IEEE VTS Japan in 2010 and Student Paper Award from IEEE Kansai Section in 2011. 